\title{
Óleo essencial de orégano: interferência da composição química na atividade frente a Salmonella Enteritidis
}

\author{
Oregano essential oil: influence of the chemical composition on the inhibitory \\ activity against Salmonella Enteritidis
}

\author{
Janine Passos Lima SILVA ${ }^{1 \star}$, Joaquim Maurício DUARTE-ALMEIDA², \\ Daniel Vidal PEREZ ${ }^{3}$, Bernadette Dora Gombossy de Melo FRANCO ${ }^{2}$
}

\section{Resumo}

Este trabalho avaliou a interferência da origem e do teor de compostos fenólicos de óleo essencial de orégano (OEO) de cinco marcas comerciais provenientes de diferentes regiões do mundo, na atividade inibitória frente à Salmonella Enteritidis (SE). A composição de cada OEO foi determinada por cromatografia gasosa acoplada ao espectrômetro de massas (CG-IE-MS). A atividade inibitória frente SE in vitro foi avaliada pela técnica de difusão em poços, empregando-se soluções alcoólicas a 0,$1 ; 0,2 ; 0,5 ; 1,0$; ou 2,0\%, observando-se a formação de halo de inibição após incubação a $30^{\circ} \mathrm{C}$ por 24 horas. Como resultado da análise por CG-IE-MS, foram identificados dezessete componentes voláteis. Todos $\mathrm{OEO}$ analisados neste trabalho possuíam carvacrol como componente principal e apresentaram atividade antimicrobiana frente a SE nas cinco concentrações testadas. A ação inibitória das cinco marcas comerciais avaliadas não apresentou diferença estatística significativa $(\mathrm{p}>0,05)$, porém o OEO proveniente da região do Mediterrâneo com p-cimeno e $\gamma$-terpineno, além de carvacrol, apresentou maiores halos de inibição de SE que os demais OEO. Concluiu-se que a multiplicação de Salmonella Enteritidis in vitro pode ser inibida por OEO cuja ação antimicrobiana independe da região produtora de orégano. No entanto, óleos essenciais que possuem p-cimeno e $\gamma$-terpineno, além de carvacrol, podem ter o seu efeito antimicrobiano potencializado.

Palavras-chave: óleo essencial de orégano; composição química; compostos fenólicos; Salmonella Enteritidis; antimicrobianos naturais.

\begin{abstract}
This study evaluated the influence of the origin and content of phenolic compounds in five Oregano Essential Oil (OEO) brands, from different part of the world, on the inhibitory activity against Salmonella Enteritidis (SE). The composition of each OEO was determined by gas chromatography coupled to mass spectrometry (GC-MS-IE). The inhibitory activity on SE was evaluated by the agar well diffusion test using alcoholic solutions at $0.1,0.2,0.5,1.0$, or $2.0 \%$, observing the formation of inhibition halos after 24 hours of incubation at $30^{\circ} \mathrm{C}$. Using a GC-MS-IE seventeen volatile components were identified. Carvacrol was the major component of all OEO samples. They were active against $\mathrm{SE}$ in all tested concentrations. The differences in the inhibitory activity presented by the five brands were not significant ( $\mathrm{p}>0.05$ ), but the OEO from the Mediterranean, which contained p-cymene and $\gamma$-terpinene besides carvacrol, resulted in larger inhibition halos than the other OEO. It was concluded that the in vitro growth of Salmonella Enteritidis can be inhibited by OEO, regardless the region in which oregano is produced. However, the essential oils that contain carvacrol, p- cymene, and $\gamma$-terpinene may present a more effective antimicrobial effect. Keywords: oregano essential oil; chemical composition; phenolic compouds; Salmonella Enteritidis; natural antimicrobials.
\end{abstract}

\section{Introdução}

As doenças transmitidas por alimentos contaminados com microrganismos patogênicos são constante motivo de preocupação para a indústria alimentícia, consumidores e agentes fiscalizadores de todo o mundo. A salmonelose, causada por Salmonella spp., é a principal dessas doenças, ocupando posição destacada no campo da saúde pública pelas suas características de morbidade e, em particular, pela dificuldade de seu controle. O sorovar Salmonella Enteritidis (SE) está envolvido em grande parte dos surtos por salmonelose. Segundo dados do Instituto Adolfo Lutz, entre janeiro e junho de 1995, o SE correspondeu a $43,4 \%$ dos sorovares de Salmonella spp. isolados de material não humano (principalmente em aves e ovos). Esse fato leva as indústrias de alimentos a buscarem alternativas tecnológicas para reduzir ou eliminar SE de seus produtos. $\mathrm{O}$ uso de antimicrobianos naturais, como temperos, condimentos e extratos vegetais, tende a ser uma alternativa interessante, principalmente quando empregado em combinação com outras tecnologias já existentes (BURT, 2004; ISAACS et al., 2005; NAZER et al., 2005; DUPONT et al., 2006).

Os componentes bioativos desses condimentos têm atividade antimicrobiana bem documentada, com atividade

Recebido para publicação em 16/6/2008

Aceito para publicação em 16/5/2009 (003428)

Departamento de Alimentos e Nutrição Experimental, Faculdade de Ciências Farmacêuticas, Embrapa Agroindústria de Alimentos, Universidade de São Paulo - USP, Av. das Américas, CP 29501, CEP 23020-470, Rio de Janeiro - RJ, Brasil

${ }^{2}$ Departamento de Alimentos e Nutrição Experimental, Faculdade de Ciências Farmacêuticas, Universidade de São Paulo - USP, Av. Prof. Lineu Prestes 580, CEP 05508-900, São Paulo - SP, Brasil, E-mail: janine@ctaa.embrapa.br

${ }^{3}$ Embrapa Solos, Rua Jardim Botânico, 1024, CEP 22460-000, Rio de Janeiro - RJ, Brasil

${ }^{*}$ A quem a correspondência deve ser enviada 
inibitória contra bactérias Gram positivas e Gram negativas, inclusive SE (LIS-BALCHIN et al., 1998; DELAQUIS et al., 2002; SAGDIÇ; ÖZCAN, 2003, NAZER et al., 2005, SEYDIM; SARIKUS, 2006).

Os óleos essenciais de condimentos podem ter muitos componentes, sendo os compostos fenólicos os principais responsáveis pelas propriedades antimicrobianas. Os compostos fenólicos são hidrofóbicos e o seu sítio de ação é a membrana celular da célula microbiana. Esses compostos se acumulam na bicamada lipídica causando desarranjo na função e na estrutura da membrana e penetram a célula bacteriana, exercendo atividade inibitória no citoplasma celular, provocando lise e liberação do ATP intracelular (WALSH et al., 2003). Outro mecanismo documentado é a perda de constituintes celulares pelo aumento da permeabilidade da membrana citoplasmática (Smith-Palmer; STEWART; FYFE, 1998; CONSENTINO et al., 1999; POL; SMID, 1999; FRIEDMAN; HENIKA; MANDRELL, 2002; Rhayour et al., 2003; YUSTE; FUNG, 2003, NAZER et al., 2005).

Os principais componentes antimicrobianos presentes no óleo essencial de orégano (OEO) são o carvacrol e o timol com comprovado efeito inibitório no controle in vitro da multiplicação de Salmonella (RHAYOUR et al., 2003; NAZER et al., 2005; SILVA et al., 2005). No entanto, a eficácia da atividade bactericida de $\mathrm{OEO}$ pode variar em função dos teores de timol e carvacrol presentes, que dependem de fatores abióticos como tipo de solo, clima, práticas agrícolas, variedade do orégano e processo de extração do óleo essencial (CONSENTINO et al., 1999; JULIANO; MATTANA; USAI, 2000; FALEIRO et al., 2002; FALCONE et al., 2005; OUSSALAH et al., 2006).

A proposta deste trabalho foi avaliar a interferência da origem do $\mathrm{OEO}$ e, consequentemente, do teor de compostos fenólicos presentes nesses produtos, na atividade inibitória frente a SE.

\section{Material e métodos}

\subsection{Cultura microbiana}

Salmonella enterica subespécie enterica sorovar Enteritidis ATCC 13076 (SE), mantida em ágar soja tripticaseína (TSA) a $4{ }^{\circ} \mathrm{C}$, reativada em caldo infusão de cérebro e coração $(\mathrm{BHI})$ a $30{ }^{\circ} \mathrm{C}$ por 18 horas.

\section{2 Óleo essencial}

Foram preparadas, no momento da realização de cada experimento, soluções com as concentrações de $0,0 \%$ (controle) 0,$1 ; 0,2 ; 0,5 ; 1,0 ;$ ou $2,0 \%(\mathrm{v} / \mathrm{v})$ de OEO de cinco marcas comerciais diferentes $(\mathrm{A}, \mathrm{B}, \mathrm{C}, \mathrm{D}, \mathrm{E})$, utilizando como solvente álcool 95\% P.A.

\subsection{Avaliação da composição química de OEO das marcas $A, B, C, D, E$}

Utilizou-se a cromatografia gasosa com detecção por espectrometria de massas com ionização por impacto de elétrons (CG-IE-MS). Para a identificação dos constituintes de cada um dos cinco óleos essenciais, procedeu-se à diluição das amostras com hexano, e $1 \mu \mathrm{L}$ de cada amostra foi injetada automaticamente (injetor HP ALS-1100 - Hewlett Packard, Palo Alto, EUA) em modo split (100:1). A separação e a análise dos componentes foram realizadas em cromatógrafo gasoso HP 5890 ser. II Plus acoplado a espectrômetro de massas HP 5973 MSD, utilizando ionização por impacto de elétrons a $70 \mathrm{eV}$ monitorado pelo software ChemStation. Como gás de arraste foi utilizado Hélio, com fluxo constante de $1 \mathrm{~cm}^{3} /$ minuto. Foi utilizada uma coluna capilar de sílica fundida HP-5 $(30 \mathrm{~m} \times 0,32 \mathrm{~mm} \times 0,25 \mu \mathrm{m})$. As temperaturas utilizadas foram de $250^{\circ} \mathrm{C}$ para o injetor e de $250^{\circ} \mathrm{C}$ para a interface entre o cromatógrafo e o detector. A temperatura inicial da coluna foi de $40^{\circ} \mathrm{C}$ durante 2 minutos, elevando-se $5^{\circ} \mathrm{C} /$ minuto até $90{ }^{\circ} \mathrm{C}$ e, posteriormente, $10{ }^{\circ} \mathrm{C} /$ minuto até $250{ }^{\circ} \mathrm{C}$. Essa temperatura foi mantida por mais 5 minutos, totalizando 35 minutos de corrida.

\subsection{Avaliação do efeito antimicrobiano}

A sensibilidade da cepa de SE à ação do OEO foi avaliada pelo método de difusão em poços descrito por Harris et al. (1989). Em placas de Petri, foram realizadas semeaduras de profundidade com cultura de SE contendo $10^{4} \mathrm{UFC} . \mathrm{mL}^{-1}$ preparada conforme descrito no item 2.1 - e caldo triptona de soja com $1 \%$ de ágar (TSBA). Após o meio solidificar, poços de $6 \mathrm{~mm}$ foram perfurados e preenchidos com $35 \mu \mathrm{L}$ de cada solução descrita no item 2.2. Após incubação a $30^{\circ} \mathrm{C}$ por 24 horas, o diâmetro dos halos de inibição de cada poço foi medido com o auxílio de uma régua de leitura de antibiograma, em que o tamanho do halo era diretamente proporcional à atividade antimicrobiana. Nessa avaliação foi utilizado um controle negativo do solvente do OEO (álcool 95\% P.A.).

\subsection{Análise estatística}

Os resultados experimentais foram submetidos à análise de variância (ANOVA) e ao teste de médias pelo programa Sisvar para Windows versão 4.0 ( $\mathrm{p}<0,05)$ (FERREIRA, 2000). Os dados da ANOVA também foram submetidos ao teste de Tukey, empregando-se o pacote estatístico SAS (1992).

\section{Resultados e discussão}

Dezessete componentes voláteis foram detectados nos $\mathrm{OEO}$ comerciais analisados por CG-IE-MS. A Figura 1 mostra os cromatogramas dos cinco OEO.

Todos os cromatogramas apresentaram um único grande pico em um mesmo tempo de retenção que, comparado a um padrão, foi identificado como carvacrol (tempo de retenção de 14 minutos), indicando que todos os OEO analisados continham carvacrol como principal componente, com variação entre 61,7 e $93,4 \%$ do total de voláteis detectados em cada óleo. Os picos dos cromatogramas estão identificados na Figura 2, onde é possível observar que o timol foi o segundo principal componente, depois do carvacrol. O timol esteve presente em três dos cinco OEO analisados (marcas A, B e C) com teores de 14,79\% na marca A, $23,85 \%$ na marca B e $1,88 \%$ na marca $C$. 

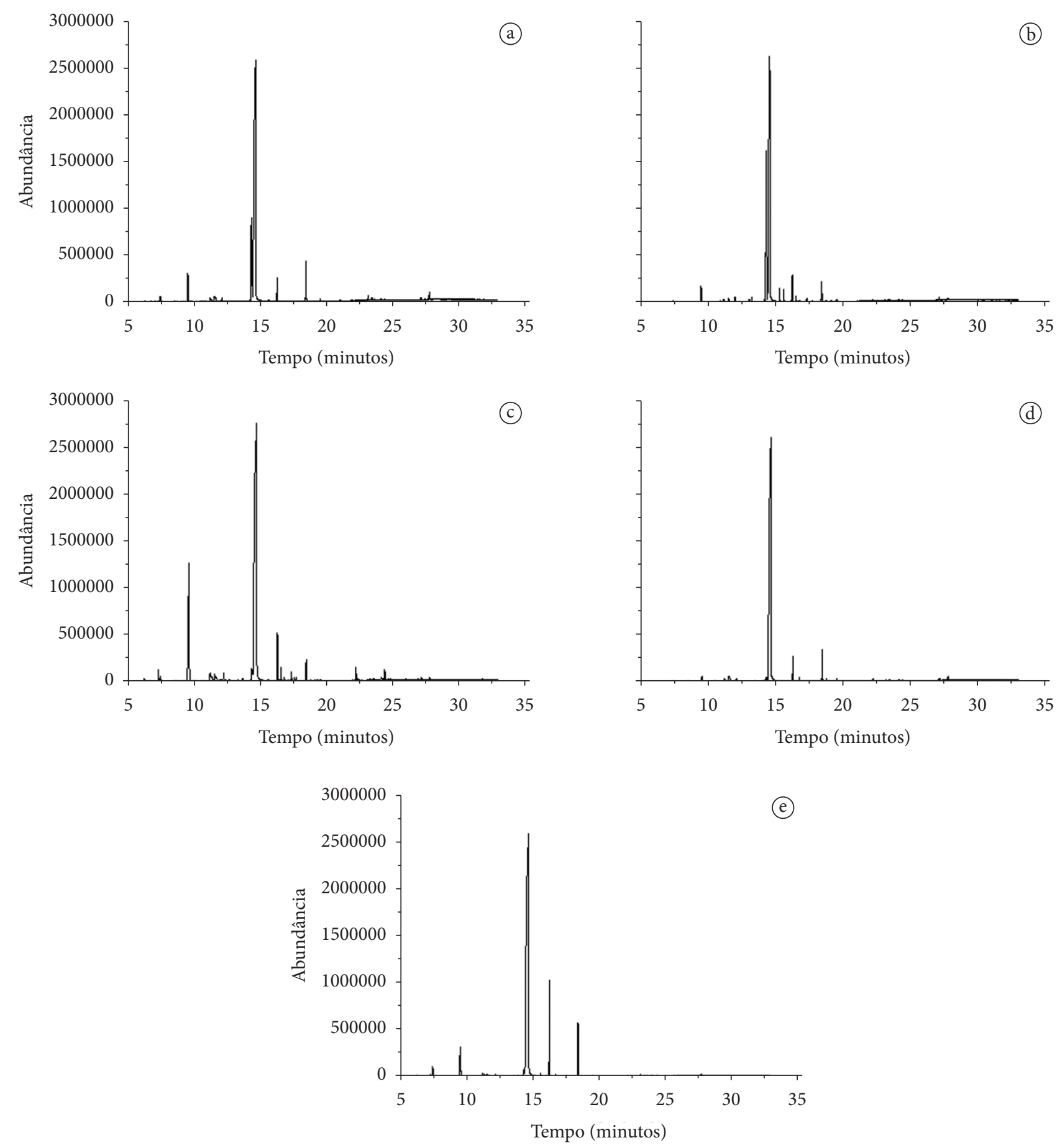

Figura 1. Cromatogramas dos OEO das marcas A, B, C, D, E.

Os teores dos quatro principais componentes encontrados nos OEO das cinco marcas estudadas, determinados através da análise por GC-IE-MS, estão descritos na Tabela 1.

Os resultados de composição dos OEO estão de acordo com aqueles encontrados por vários pesquisadores que analisaram os componentes de óleos essenciais de orégano por CG-IE-MS. Kokkini et al. (1997) coletaram orégano da região noroeste e sudeste da Grécia e observaram a presença dominante de
Tabela 1. Teores de carvacrol, $\gamma$-terpineno, p-cimeno e timol em óleo essencial de orégano de cinco diferentes marcas (A, B, C, D e E).

\begin{tabular}{lccccc}
\hline Componente & \multicolumn{6}{c}{ Teor (\%) por marca } \\
\hline & $\mathrm{A}$ & $\mathrm{B}$ & $\mathrm{C}$ & $\mathrm{D}$ & $\mathrm{E}$ \\
\hline Carvacrol & 74,45 & 67,73 & 72,61 & 93,42 & 61,66 \\
Gama Terpineno & - & - & - & - & 6,90 \\
p-Cimeno & - & - & 0,63 & - & 15,95 \\
Timol & 14,79 & 23,85 & 1,88 & - & - \\
\hline
\end{tabular}


carvacrol nas amostras provenientes do noroeste e timol nas do sudeste, para uma mesma época do ano (outono ou verão). No entanto, em ambas as marcas, o teor de carvacrol sempre era maior no verão, uma vez que o teor dos compostos fenólicos aumenta na florescência. Outros pesquisadores também constataram que carvacrol e timol são os principais constituintes e os responsáveis pela atividade antimicrobiana de OEO (SIVROPOULOU et al., 1996; LAMBERT et al., 2001; CHI; ZIVANOVIC; PENFIELD, 2006). Além disso, há evidências de que alguns componentes presentes em menor quantidade que os compostos fenólicos, como $\gamma$-terpineno e p-cimeno, interferem na atividade antimicrobiana por produzirem efeito sinérgico entre os demais componentes (PASTER et al., 1995).

Na Figura 2, também pode ser observado que $\gamma$-terpineno e p-cimeno (monoterpenos precursores do carvacrol) são constituintes secundários e presentes em menor quantidade no óleo da marca E, proveniente da região do Mediterrâneo. Muitos pesquisadores que analisaram orégano procedente dessa região

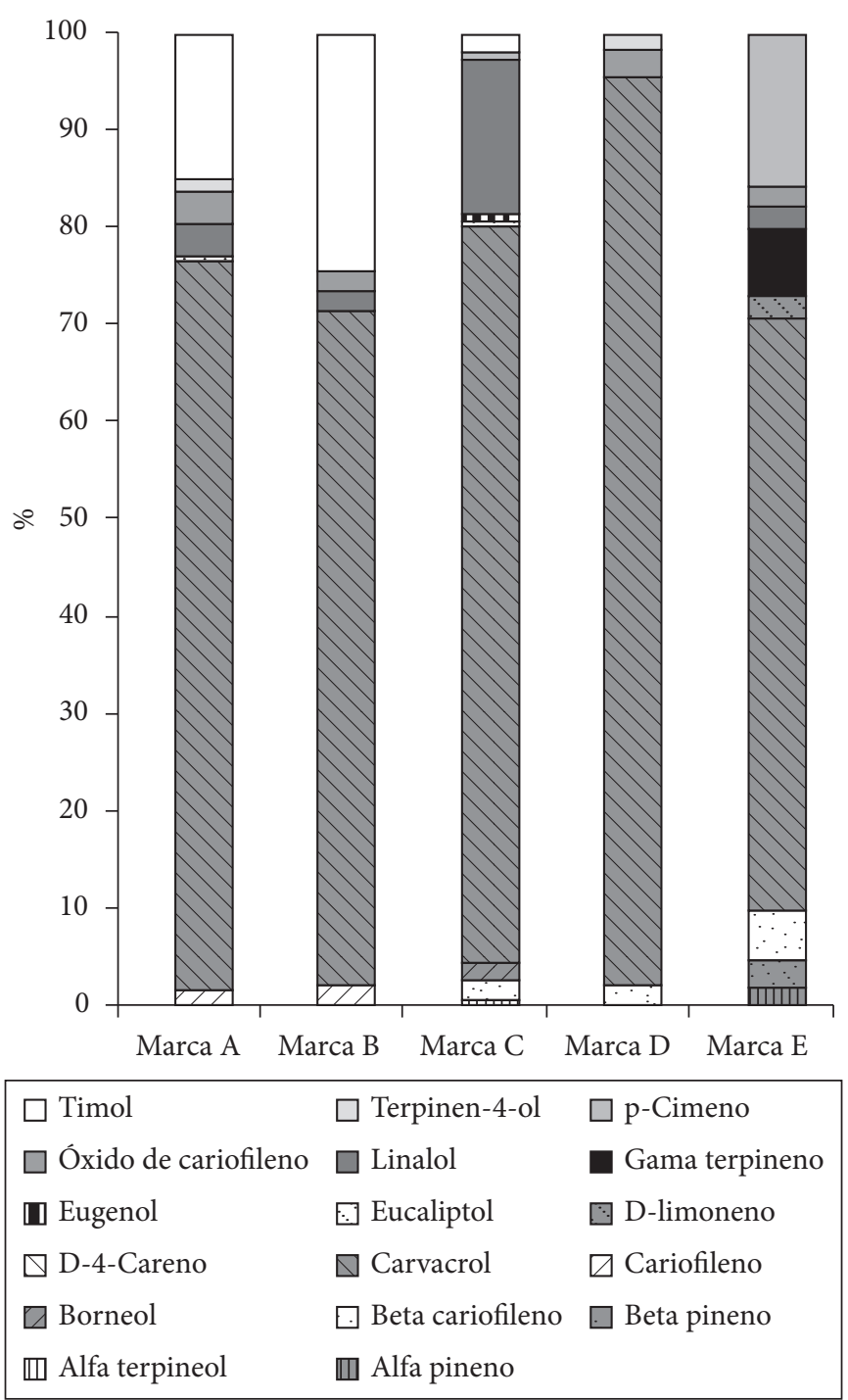

Figura 2. Composição química dos OEO das marcas A, B, C, D, E. encontraram resultados semelhantes (SIVROPOULOU et al., 1996, CHORIANOPOULOS et al., 2004). Kokkini et al. (1997) verificaram que, no orégano (Origanum vulgare subsp. hirtum) proveniente de três regiões geográficas diferentes na Grécia, a soma do teor dos quatro componentes principais foi semelhante nas plantas colhidas no verão e no outono. No entanto, esses autores observaram também que o teor de cada componente no orégano colhido em uma mesma região variou em relação à época do ano. No verão, o teor de p-cimeno foi menor, independente da região. A quantidade de carvacrol no orégano das três regiões estudadas variou independentemente da época da colheita: em uma das regiões, o teor de carvacrol ficou entre 1,7 e 2,5\%, enquanto nas outras duas as concentrações variaram de 57,4 a $69,6 \%$. O inverso aconteceu com o timol (CHORIANOPOULOS et al., 2004; DAFERERA; ZIOGAS; POLISSIOU, 2003; KOKKINI et al., 1997; SIVROPOULOU et al., 1996).

Geralmente os óleos essenciais extraídos de plantas colhidas durante ou imediatamente após o florescimento possuem atividade antimicrobiana mais intensa (MARINO; BERSANI; COMI, 1999). O orégano cultivado no sul da Croácia, colhido durante cinco meses entre abril e agosto, apresentou variações nos teores de 28 componentes identificados, apresentando a maior concentração de todos eles no período de florescimento em julho, com um significante decréscimo em agosto (JERKOVIC; MASTELIC; MILOS, 2001). No orégano croata, o teor de timol foi mais elevado que o de carvacrol, sendo similar ao orégano de uma das regiões da Grécia reportado por Kokkini et al. (1997).

Com relação à atividade antimicrobiana dos OEO estudados, os resultados indicaram que todas as cinco marcas (A, B, C, D e E) apresentaram atividade antimicrobiana frente a SE. Pode-se observar na Tabela 2 que o aumento do tamanho do halo de inibição, medido em milímetros, foi proporcional à concentração do OEO de 0,1 até $2,0 \%$.

A análise estatística dos resultados indicou que os OEO pertencentes às marcas $\mathrm{A}, \mathrm{B}, \mathrm{D}$ e E tiveram desempenho semelhante para uma mesma concentração testada, com exceção apenas daquele pertencente à marca $\mathrm{C}$ na concentração de $0,1 \%$, que resultou em halo de inibição significativamente menor que os demais OEO nessa concentração $(\mathrm{p}<0,05)$. Considerando todas as concentrações testadas, a análise estatística demonstrou que não houve diferença significativa na atividade inibitória in vitro dos cinco OEO testados ( $\mathrm{p}<0,05)$. Note-se, no entanto, que o OEO da marca E, extraído de orégano cultivado na região do Mediterrâneo, apresentou halos ligeiramente maiores do que os demais óleos na concentração $\geq 0,2 \%$. Esse fato pode ser decorrente da presença de p-cimeno e $\gamma$-terpineno no OEO da marca E. Esse resultado está de acordo com Chi, Zivanovic e Penfiled (2006) que também observaram que a atividade inibitória do óleo essencial de orégano varia de acordo com a sua composição.

Conforme pode ser visto na Tabela 2, não houve diferença significativa no tamanho dos halos decorrentes do uso de OEO das marcas A, D e E nas concentrações de 0,2 e 0,5\%. 
Tabela 2. Atividade inibitória medida pelo tamanho do halo de diferentes concentrações de óleo essencial de orégano (OEO) de cinco marcas (A, B, C, D e E) frente a Salmonella Enteritidis a $30^{\circ} \mathrm{C}$ determinada pela técnica de difusão em poços.

\begin{tabular}{|c|c|c|c|c|c|}
\hline \multirow[b]{2}{*}{ OEO (\%) } & \multicolumn{5}{|c|}{ 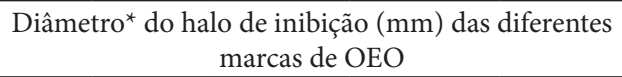 } \\
\hline & A & B & $\mathrm{C}$ & $\mathrm{D}$ & $\mathrm{E}$ \\
\hline 0,1 & $12^{\mathrm{A}, \mathrm{d}}$ & $12^{\mathrm{A}, \mathrm{c}}$ & $7^{\mathrm{B}, \mathrm{e}}$ & $10^{\mathrm{A}, \mathrm{d}}$ & $11^{\mathrm{A}, \mathrm{d}}$ \\
\hline 0,2 & $14^{\mathrm{A}, \mathrm{c}}$ & $13^{\mathrm{A}, \mathrm{c}}$ & $13^{\mathrm{A}, \mathrm{d}}$ & $13^{\mathrm{A}, \mathrm{c}}$ & $15^{\mathrm{A}, \mathrm{c}}$ \\
\hline 0,5 & $15^{\mathrm{A}, \mathrm{c}}$ & $14^{\mathrm{A}, \mathrm{b}}$ & $15^{\mathrm{A}, \mathrm{c}}$ & $15^{\mathrm{A}, \mathrm{c}}$ & $16^{\mathrm{A}, \mathrm{c}}$ \\
\hline 1,0 & $18^{\mathrm{A}, \mathrm{b}}$ & $16^{\mathrm{A}, \mathrm{b}}$ & $18^{\mathrm{A}, \mathrm{b}}$ & $18^{\mathrm{A}, \mathrm{b}}$ & $19^{\mathrm{A}, \mathrm{b}}$ \\
\hline 2,0 & $21^{\mathrm{A}, \mathrm{a}}$ & $20^{A, a}$ & $22^{\mathrm{A}, \mathrm{a}}$ & $21^{\mathrm{A}, \mathrm{a}}$ & $22^{\mathrm{A}, \mathrm{a}}$ \\
\hline \multicolumn{6}{|c|}{$\begin{array}{l}\text { *o diâmetro do halo de inibição inclui } 6 \mathrm{~mm} \text { do poço perfurado. }{ }^{* *} \text { cada valor é a } \\
\text { média de nove medições; } \mathrm{e}^{* * *} \text { para uma mesma linha, valores seguidos da mesma letra } \\
\text { maiúscula não aparesentaram diferença estatística entre } \mathrm{si}(\mathrm{p}<0,05) \text { para uma mesma } \\
\text { coluna, valores seguidos da mesma letra minúscula não apresentaram diferença estatística } \\
\text { entre si }(\mathrm{p}<0,05) \text {. }\end{array}$} \\
\hline
\end{tabular}

Os OEO das marcas A e B, que contém timol, na concentração de $0,1 \%$ apresentaram halos de inibição de SE maiores do que os óleos que não possuem timol. Outros autores também observaram que as bactérias Gram negativas são mais sensíveis ao timol do que ao carvacrol (SIVROPOULOU et al., 1996, Chorianopoulos et al., 2004). Entretanto, esses autores comprovaram que as bactérias Gram positivas são mais sensíveis à ação de $\mathrm{OEO}$, independente do teor de timol ou carvacrol, do que as Gram negativas.

Chorianopoulos et al. (2004) afirmaram que a atividade antimicrobiana não se deve somente à presença de carvacrol e timol, mas que a presença de outros componentes em baixas concentrações pode provocar interações sinérgicas, aditivas ou antagônicas. Baydar et al. (2004) testaram um OEO com 53\% de carvacrol e timol e outro OEO com $87 \%$ desses mesmos componentes e, como resultado, verificaram que o primeiro OEO, mesmo tendo menos carvacrol e timol, apresentou uma ação antimicrobiana mais efetiva por causa da presença de p-cimeno e $\gamma$-terpineno. Segundo Ultee et al. (2000), o p-cimeno não tem efeito antibacteriano se usado sozinho, mas, quando combinado com carvacrol, ocorre sinergismo entre os dois. Neste trabalho, entre os cinco OEO avaliados, o óleo da marca E foi o que apresentou o menor teor de carvacrol $(61,7 \%)$ e os maiores halos de inibição a partir da concentração $0,2 \%$. Possivelmente isso ocorreu devido à presença de $\mathrm{p}$-cimeno $\mathrm{e}$ também de $\gamma$-terpineno nesse óleo. Já o OEO da marca $D$, que tinha o teor mais elevado de carvacrol $(93,4 \%)$, mas não tinha p-cimeno ou $\gamma$-terpineno, apresentou halos menores que os do OEO da marca E. Segundo Burt (2004), o p-cimeno facilita o transporte do carvacrol através da membrana citoplasmática para o interior da célula bacteriana, o que provavelmente não ocorreu de forma efetiva com o carvacrol do OEO da marca D por causa da ausência de p-cimeno.

\section{Conclusões}

A multiplicação de Salmonella Enteritidis pode ser inibida por OEO cuja ação antimicrobiana aparentemente independe da região produtora de orégano ou da época do ano. No entanto, óleos essenciais de orégano que possuem p-cimeno, gama- terpineno e carvacrol podem ter o seu efeito antimicrobiano frente a SE potencializado.

\section{Agradecimentos}

Ao $\mathrm{CNPq} / \mathrm{MESA}$ pelo apoio financeiro.

\section{Referências bibliográficas}

BAYDAR, H. et al. Antibacterial activity and composition of essential oils from Origanum, Thymbra and Satureja species with commercial importance in Turkey. Food Control, v. 15, n. 3, p. 169-172, 2004.

BURT, S. Essential oils: their antibacterial properties and potential applications in foods-a review. International Journal of Food Microbiology, v. 94, n. 3, p. 223-253, 2004.

CHI, S.; ZIVANOVIC, S.; PENFIELD, M. P. Application of chitosan films enriched with oregano essential oil on bologna- active compounds and sensory attributes. Food Science and Technology International, v. 12, n. 2, p. 111-117, 2006.

CHORIANOPOULOS, N. et al. Essential oils of satureja, origanum, and thymus species: chemical composition and antibacterial activities against foodborne pathogens. Journal of Agricultural and Food Chemistry, v. 52, n. 26, p. 8261-8267, 2004.

CONSENTINO, S. et al. In vitro antimicrobial activity and chemical composition of Sardinian Thymus essential oils. Letters in Applied Microbiology, v. 29, n. 2, p. 130-135, 1999.

DAFERERA, D. J.; ZIOGAS, B. N.; POLISSIOU, M. G. The effectiveness of plant essential oils in the growth of Botrytis cinerea, Fusarium sp. and Clavibacter michiganensis subsp. michiganensis. Crop Protection, v. 22, n. 1, p. 39-44, 2003.

DELAQUIS, P. J. et al. Antimicrobial activity of individual and mixed fractions of dill, cilantro, coriander and eucalyptus essential oils. International Journal of Food Microbiology, v. 74, n. 1, p. 101-109, 2002.

DUPONT, S. et al. In vitro antibacterial activity of Australian native herb extracts against food-related bacteria. Food Control, v. 17, n. 11, p. 929-932, 2006.

FALCONE, P. et al. A study on the antimicrobial activity of thymol intended as a natural preservative. Journal of Food Protection, v. 68, n. 8, p. 1664-1670, 2005.

FALEIRO, M. L. et al. Antimicrobial activity of essential oils isolated from Portuguese endemic species of thymus. Letters in Applied Microbiology, v. 36, n. 1, p. 35-40, 2003.

FERREIRA, D. F. Análises estatísticas por meio do Sisvar para Windows versão 4.0. In: Reunião Anual da Região Brasileira da Sociedade internacional de Biometria, 45., 2000, São Carlos. Anais... São Carlos: UFSCar, 2000. p. 255-258.

FRIEDMAN, M.; HENIKA, P. R.; MANDRELL, R. E. Bactericidal activities of plant essential oils and some of their isolated constituents against Campylobacter jejuni, Escherichia coli, Listeria monocytogenes, and Salmonella enterica. Journal of Food Protection, v. 65, n. 10, p. 1545-1560, 2002.

HARRIS, L. J. et al. Antimicrobial activity of lactic acid bacteria against Listeria monocytogenes. Journal of Food Protection, v. 52, n. 6, p. 384-387, 1989.

ISAACS, S. et al. An International Outbreak of Salmonellosis Associated with Raw Almonds Contaminated with a Rare Phage Type of Salmonella Enteritidis. Journal of Food Protection, v. 68, n. 1, p. 191-198, 2005. 
JERKOVIC, I.; MASTELIC, J.; MILOS, M. The impact of both the season of collection and drying on the volatile constituents of Origanum vulgare L. ssp. hirtum grown wild in Croatia. International Journal of Food Science and Technology, v. 36, n. 6, p. 649- 654, 2001.

JULIANO, C.; MATTANA, A.; USAI, M. Composition and in vitro antimicrobial activity of the essential oil of Thymus herba-barona Loisel growing wild in Sardinia. Journal of Essential Oil Research, v. 12 , n. 4 , p. $516-522,2000$.

KOKKINI, S. et al. Autumn essential oils of Greek oregano. Phytochemistry, v. 44, n. 5, p. 883-886, 1997.

LAMBERT, R. J. W. et al. A study of the minimum inhibitory concentration and mode of action of oregano essential oil, thymol and carvacrol. Journal of Applied Microbiology, v. 91, n. 3, p. 453-462, 2001.

LIS-BALCHIN, M. et al. Antimicrobial activity of Pelargonium essential oils added to a quiche filling as a model food system. Letters in Applied Microbiology, v. 27, n. 4, p. 207-210, 1998.

MARINO, M.; BERSANI, C.; COMI, G. Antimicrobial activity of the essential oils of Thymus vulgaris L. measured using a bioimpedometric method. Journal of Food Protection, v. 62, n. 9 , p. 1017-1023, 1999.

NAZER, A. I. et al. Combinations of food antimicrobials at low levels to inhibit the growth of Salmonella sv. Typhimurium: a synergistic effect? Food Microbiology, v. 22, n. 4, p. 391-398, 2005.

OUSSALAH, M. et al. Antimicrobial effects of selected plant essential oils on the growth of a Pseudomonas putida strain isolated from meat. Meat Science, v. 73, n. 2, p. 236-244, 2006.

PASTER, N. et al. Antifungal activity of oregano and thyme essential oils applied as fumigants against fungi attacking stored grain. Journal of Food Protection, v. 58, n. 1, p. 81-85, 1995.
POL, I. E.; SMID, E. J. Combined action of nisin and carvacrol on Bacillus cereus e Listeria monocytogenes. Letters in Applied Microbiology, v. 29, n. 3, p. 166-170, 1999.

RHAYOUR, K. et al. The mechanism of bactericidal action of oregano and clove essential oils and of their phenolic major components on Escherchia coli and Bacillus subtilis. Journal of Essential Oil Research, v. 15, n. 5, p. 356-362, 2003.

SAGDIÇ, O.; ÖZCAN, M. Antibacterial activity of Turkish spice hydrosols Food Control, v. 14, n. 3, p. 141-143, 2003.

SEYDIM, A. C.; SARIKUS, G. Antimicrobial activity of whey protein based edible films incorporated with oregano, rosemary and garlic essential oils. Food Research International, v. 39, n. 5, p. 639-644, 2006.

SILVA, J. P. L. et al. Effects of oregano essential oil and nisin on growth of gram positive and gram negative foodborne pathogens. In: INTERNATIONAL ASSOCIATION FOR FOOD PROTECTION ANNUAL MEETING, 92., 2005, Baltimore. Abstracts...

SIVROPOUlOU, A. et al. Antimicrobial and cytotoxic activities of Origanum essential oils. Journal of Agricultural and Food Chemistry, v. 44, n. 5, p. 1202-1205, 1996.

SMITH-PALMER, A.; STEWART, J.; FYFE, L. Antimicrobial properties of plant essencial oils and essences against five important foodborne pathogens. Letters in Applied Microbiology, v. 26, n. 2, p. 118-122, 1998.

ULTEE, A. et al. Antimicrobial activity of carvacrol toward Bacillus cereus on rice. Journal of Food Protection, v. 63, n. 5, p. 620-624, 2000.

YUSTE, J.; FUNG, D. Y. C. Evaluation of Salmonella typhimurium, Yersinia enterocolitica and Staphylococcus aureus counts in apple juice with cinnamon, by conventional media and thin agar layer method. Food Microbiology, v. 20, n. 3, p. 365-370, 2003. 